\title{
Polarized x-ray spectroscopy of quaternary ferromagnetic semiconductor (Ga,Mn)(As,P)
}

\author{
P. Wadley ${ }^{1}$, A. Casiraghi ${ }^{1}$, M. Wang ${ }^{1}$, K.W. Edmonds ${ }^{1}$, R.P. Campion ${ }^{1}$, A.W. Rushforth ${ }^{1}$, B.L. \\ Gallagher $^{1}$, C.R. Staddon ${ }^{1}$, G. van der Laan ${ }^{2}$, E. Arenholz ${ }^{3}$ \\ ${ }^{1}$ School of Physics and Astronomy, University of Nottingham, Nottingham NG7 2RD, United Kingdom \\ ${ }^{2}$ Diamond Light Source, Chilton, Didcot, Oxfordshire OX11 ODE, United Kingdom \\ ${ }^{3}$ Advanced Light Source, Lawrence Berkeley National Laboratory, Berkeley, California 94720, USA
}

$X$-ray magnetic circular dichroism $(X M C D)$ at the $M n L_{2,3} x$-ray absorption edges is used study the magnetic and electronic properties of the quaternary diluted magnetic semiconductor $(G a, M n)(A s, P)$ as a function of $P$ concentration $y$. A clear signature of the variation in strain, from compressive to tensile on increasing $y$, is observed in the angular dependence of the hybridized $d^{5}$-like XMCD spectra. The ferromagnetic transition temperature and the magnetic moment per Mn ion both decrease steadily with increasing $y$.

Epitaxial strain is increasingly important in semiconductor microelectronics devices, enabling the growth of new combinations of materials in layered structures, and the tuning of band offsets and carrier mobility for specific applications. In diluted magnetic semiconductors such as $(\mathrm{Ga}, \mathrm{Mn}) \mathrm{As}$, which have a relatively low magnetization so that shape anisotropy is weak, strain largely determines the direction of the easy magnetic axis. Several studies have demonstrated that the magnetization direction can be controlled using strain, for example by piezoelectric gating $\left[{ }^{1}\right]$, growth on strain-relaxed $(\mathrm{In}, \mathrm{Ga}) \mathrm{As}$ buffer layers $\left[^{2}\right]$, or control of alloy composition $\left[{ }^{3},{ }^{4}\right]$. Of these approaches, alloying $(\mathrm{Ga}, \mathrm{Mn}) \mathrm{As}$ with $\mathrm{GaP}$ allows the tuning of strain across a wide range, from compressive to strongly tensile, while also avoiding the large defect densities that necessarily result from strain relaxation $\left.{ }^{5}\right]$. (Ga,Mn)(As,P) films show favorable micromagnetic properties for studies of field- and current-driven magnetic domain wall motion $\left[{ }^{6}, 7\right]$. In addition, the incorporation of $\mathrm{P}$ results in a modification of the band structure of the ferromagnetic semiconductor, influencing its electrical properties and ferromagnetic transition temperature $\left(T_{\mathrm{C}}\right)$. However, there is disagreement between several theoretic and experimental studies as to whether $T_{\mathrm{C}}$ should increase $\left[{ }^{8}\right]$ or rapidly decrease $\left[{ }^{9}\right]$ with increasing $\mathrm{P}$.

$\mathrm{X}$-ray magnetic circular dichroism (XMCD), a powerful tool for combined studies of magnetism and electronic structure, has recently been shown to be a sensitive probe of epitaxial strain in semiconducting and insulating magnetic materials $\left[{ }^{10},{ }^{11}\right]$. It thus has potential to offer unique insights into the interplay of strain, doping, band alignment and ferromagnetism in quaternary diluted magnetic semiconductors such as $(\mathrm{Ga}, \mathrm{Mn})(\mathrm{As}, \mathrm{P})$. In this paper, we present an XMCD study of a series of $(\mathrm{Ga}, \mathrm{Mn})(\mathrm{As}, \mathrm{P})$ films with varying $\mathrm{P}$ concentration.

The studied $25 \mathrm{~nm}$ thick $\mathrm{Ga}_{1-x} \mathrm{Mn}_{x} \mathrm{As}_{1-y} \mathrm{P}_{y}$ films were grown on $\mathrm{GaAs}(001)$ substrates by molecular beam epitaxy $\left[{ }^{4}\right]$. The $\mathrm{P}$ concentration $y$ was varied from 0 to $\sim 30 \%$, while the incident Mn flux was fixed to give a nominal Mn concentration $x$ of $6 \%$. The substrate temperature during growth of the $(\mathrm{Ga}, \mathrm{Mn})(\mathrm{As}, \mathrm{P})$ layer was held at $230^{\circ} \mathrm{C}$. Post-growth, the samples were annealed in air at $190^{\circ} \mathrm{C}$ in order to out-diffuse compensating interstitial Mn defects. The $\mathrm{P}$ concentration was estimated from the lattice mismatch determined by high resolution $\mathrm{x}$-ray diffraction (XRD), assuming that the incorporation of Mn and unintentional impurities, and their influence on the lattice parameter, are independent of $y\left[{ }^{4}\right]$. The $T_{\mathrm{C}}$ was obtained from superconducting quantum interference device (SQUID) measurements of the remnant magnetization along the easy magnetic axis after field-cooling. Table I compares the values of $y$ 
estimated from the ratio of anion fluxes during growth and from the XRD measurements, $T_{\mathrm{C}}$, and epitaxial strain $\left(a_{\perp}-a_{0}\right) / a_{0}$ (where $a_{\perp}$ and $a_{0}$ are the perpendicular-to-plane lattice parameter of the film and the cubic lattice parameter of the substrate, respectively) for the studied samples before and after post-growth annealing.

The XMCD measurements were performed on line 4.0.2 of the Advanced Light Source at a sample temperature of $15 \mathrm{~K}$. Prior to the measurements, the films were $\mathrm{HCl}$-etched in order to remove Mn-rich surface oxides $\left[{ }^{12}\right]$. The $\mathrm{Mn} L_{2,3}$ edge x-ray absorption (XA) spectra were acquired in total electron yield mode. The XMCD spectra were obtained by reversing the external magnetic field of up to $0.8 \mathrm{~T}$ at each energy point in the XA spectra. Figure 1 shows representative XA and XMCD spectra, obtained with the magnetization and incoming $\mathrm{X}$-ray beam directed along the surface normal. For all $\mathrm{P}$ concentrations the spectra resemble those observed previously for both $(\mathrm{Ga}, \mathrm{Mn}) \mathrm{As}\left[{ }^{12},{ }^{13}\right]$ and $(\mathrm{Ga}, \mathrm{Mn}) \mathrm{P}\left[{ }^{14}\right]$, characteristic of a hybridized $d^{5}$-like electronic configuration with tetrahedral symmetry.

The strain dependence of the XMCD spectra is visible in the pre-edge region at around $0.6 \mathrm{eV}$ below the $L_{3}$ peak. This region is shown in detail in Fig. 2(a-g), for all 7 studied samples, and for two different angles $\theta$ of the external magnetic field and the surface nomal. The external field and the x-ray helicity vector are collinear in each case. On rotating the measurement axis from normal to grazing incidence, the height of this pre-edge peak either increases or decreases, depending on the sign of the strain. The difference between the XMCD intensity at $637.8 \mathrm{eV}$ for normal incidence $(\theta=0)$ and grazing incidence $\left(\theta=60^{\circ}\right)$ is plotted versus strain in Fig. 2h, showing an approximately linear relationship.

Inspection of Fig. 2(a-g) reveals that the pre-edge peak height at normal incidence strongly depends on the $\mathrm{P}$ concentration, while at $\theta=60^{\circ}$ it is hardly affected. In axial symmetry, for collinear magnetization and X-ray helicity, the angle dependence of the XMCD can be described by

$$
I_{\mathrm{XMCD}}=I_{0}+1 / 2 I_{2}\left(3 \cos ^{2} \theta-1\right)
$$

(Ref. 11), where $I_{0}$ and $I_{2}$ are the isotropic and anisotropic components. Therefore, the anisotropic component is very small at $\theta=60^{\circ}$ (and disappears at $\theta=54.7^{\circ}$ ), consistent with the present result. For these single-crystalline $(\mathrm{Ga}, \mathrm{Mn})(\mathrm{As}, \mathrm{P})$ films, the 'isotropic' XMCD also displays a cubic symmetry due to the tetrahedral local environment of the $\mathrm{Mn}$ in the absence of strain $\left[{ }^{15}\right]$, and moreover the pre-edge peak height depends on the free carrier density, i.e. on the character of the states at the Fermi energy [10]. The weak dependence of the pre-edge structure at $\theta=60^{\circ}$ on the $\mathrm{P}$ concentration therefore indicates that the $3 d$ local density of states around the Mn ions is similar for all the studied samples.

To extract quantitative information on the Mn $3 d$ ground state magnetic moments, we performed sum rule analysis on the XMCD spectra $\left[{ }^{16},{ }^{17}\right]$, following the procedure described in Refs. 13 and 14. A $d$ electron count of 5.1 was assumed, and a correction factor of 1.47 was applied to the spin magnetic moment to account for the mixing of $2 p_{3 / 2}$ and $2 p_{1 / 2}$ multiplet peaks. The latter was obtained by applying the sum rules to an atomic multiplet calculated spectrum for a Mn $d^{5}$ configuration with a well-defined ground state magnetic moment. Finally, the obtained magnetic moments were corrected by a factor $\mathrm{M}(2 \mathrm{~K}) / \mathrm{M}(15 \mathrm{~K})$, where $\mathrm{M}(\mathrm{T})$ is the magnetization measured by SQUID at temperature $\mathrm{T}$, in order to account for the elevated temperature of the XMCD measurements.

Figure 3 shows the obtained total $3 d$ magnetic moment per ion (i.e., the sum of spin and orbital components) versus P concentration. Large magnetic moments of around $4 \mu_{\mathrm{B}}$ per Mn are obtained for films with $y<\sim 15 \%$. However, a steady decrease of the magnetic moment is 
observed with increasing $y$. The net $3 d$ magnetic moment for the highest $\mathrm{P}$ concentrations is around $35 \%$ smaller than for the (Ga,Mn)As control sample. Since the similarity of the XMCD lineshapes indicates that the local magnetic moment of the ferromagnetic $\mathrm{Mn}$ ions is not significantly changed, the reduction of the net $3 d$ moment is ascribed to a decrease in the proportion of the incorporated $\mathrm{Mn}$ that participates in the ferromagnetism.

The $T_{\mathrm{C}}$ measured by SQUID magnetometry, also shown in Fig. 3, shows the same decreasing trend with increasing $y$. It seems reasonable to associate this reduction in $T_{\mathrm{C}}$ to the decreased proportion of ferromagnetic Mn ions. This may in turn be associated with a decrease in the ability of the charge carriers to mediate the ferromagnetic ordering $\left[{ }^{18}\right]$, either due to enhanced compensation or increased localization in the quaternary alloy system. It is also interesting to note that a quantitatively similar decrease of $T_{\mathrm{C}}$ with alloy concentration $y$ is observed in $\mathrm{Al}_{y} \mathrm{Ga}_{1-y-x} \mathrm{Mn}_{\mathrm{x}} \mathrm{As}\left[{ }^{19}\right]$, indicating that the same physical process (e.g., compensation or localization of carriers) may be responsible in both cases.

Supported by the EU (Grant nos. SemiSpinNet-215368 and NAMASTE-214499), STFC (Grant no. CMPC07100), and the U.S. DoE under Contract No. DE-AC02-05CH11231.

\begin{tabular}{|c|c|c|c|c|c|}
\hline Nominal $y(\%)$ & $\begin{array}{c}\text { Estimated } y \\
(\%)\end{array}$ & $\begin{array}{c}T_{\mathrm{C}} \text { as-grown } \\
(\mathrm{K})\end{array}$ & $\begin{array}{c}T_{\mathrm{C}} \text { annealed } \\
(\mathrm{K})\end{array}$ & $\begin{array}{c}\text { Strain as- } \\
\text { grown }(\%)\end{array}$ & $\begin{array}{c}\text { Strain annealed } \\
(\%)\end{array}$ \\
\hline 0 & 0 & 66 & 137 & -0.63 & -0.38 \\
\hline 3 & 5.5 & 61 & 124 & -0.25 & +0.01 \\
\hline 6 & 5.9 & 52 & 121 & -0.22 & +0.06 \\
\hline 9 & 9.2 & 47 & 108 & 0.00 & +0.3 \\
\hline 10 & 12.4 & 32 & 107 & +0.28 & +0.51 \\
\hline 20 & 27.6 & 18 & 78 & +1.32 & +1.54 \\
\hline 30 & 31.5 & 15 & 75 & +1.58 & +1.81 \\
\hline
\end{tabular}

Table I. Properties of the studied $(\mathrm{Ga}, \mathrm{Mn})(\mathrm{As}, \mathrm{P})$ films: nominal P concentration from flux ratios during growth; $\mathrm{P}$ concentration estimated from XRD; $T_{\mathrm{C}}$ of the as-grown and annealed films; and epitaxial strain $\left(a_{\perp}-a_{0}\right) / a_{0}$ of the as-grown and annealed films.

\footnotetext{
${ }^{1}$ M Overby, A Chernyshov, L P Rokhinson, X Liu, and J K Furdyna, Appl. Phys. Lett. 92, 192501 (2008); A W Rushforth, E De Ranieri, J Zemen, J Wunderlich, K W Edmonds, C S King, E Ahmad, R P Campion, C T Foxon, B L Gallagher, K Vyborny, J Kucera, and T Jungwirth, Phys. Rev. B 78, 085314 (2008).

${ }^{2}$ A. Shen, H. Ohno, F. Matsukura, Y. Sugawara, N. Akiba, T. Kuroiwa, A. Oiwa, A. Endo, S. Katsumoto, and Y. Iye, J. Cryst. Growth 175-176, 1069 (1997).

${ }^{3}$ P. R. Stone, J. W. Beeman, K. M. Yu, and O. D. Dubon, Physica B (Amsterdam) 401, 454 (2007); A. Miard, L. Travers, O. Mauguin, L. Largeau, C. Gourdon, V. Jeudy, M. Tran, J. -M. George, and A. Lemaître, Appl. Phys. Lett. 93, 021123 (2008); M. Cubukcu, H. J. von Bardeleben, Kh. Khazen, J. L. Cantin, O. Mauguin, L. Largeau, and A. Lemaître, Phys. Rev. B 81, 041202 (2010).

${ }^{4}$ A. W. Rushforth, M. Wang, N. R. S. Farley, R. P. Campion, K. W. Edmonds, C. R. Staddon, C. T. Foxon, and B. L. Gallagher, J. Appl. Phys. 104, 073908 (2008).

${ }^{5}$ K. Y. Wang, A. W. Rushforth, V. A. Grant, R. P. Campion, K. W. Edmonds, C. R. Staddon, C. T. Foxon, B. L. Gallagher, J. Wunderlich, and D. A. Williams, J. Appl. Phys. 101, 106101 (2007)

${ }^{6}$ S. Haghgoo, M. Cubukcu, H. J. von Bardeleben, L. Thevenard, A. Lemaître, and C. Gourdon, Phys. Rev. B 82, 041301 (2010).

${ }^{7}$ K. Y. Wang, K. W. Edmonds, A. C. Irvine, G. Tatara, E. De Ranieri, J. Wunderlich, K. Olejnik, A. W. Rushforth, R. P. Campion, D. A. Williams, C. T. Foxon and B. L. Gallagher, Appl. Phys. Lett. 97, 262102 (2010).

${ }^{8}$ J. Mašek, J. Kudrnovský, F. Máca, J. Sinova, A. H. MacDonald, R. P. Campion, B. L. Gallagher, and T. Jungwirth, Phys. Rev. B 75, 045202 (2007).
} 
${ }^{9}$ P. R. Stone, K. Alberi, S. K. Z. Tardif, J.W. Beeman, K. M. Yu, W. Walukiewicz, and O. D. Dubon, Phys. Rev. Lett. 101, 087203 (2008).

${ }^{10}$ G. van der Laan, K. W. Edmonds, E. Arenholz, N. R. S. Farley, and B. L. Gallagher, Phys. Rev. B 81, 214422 (2010).

${ }^{11}$ G. van der Laan, R. V. Chopdekar, Y. Suzuki, and E. Arenholz, Phys. Rev. Lett. 105, 067405 (2010).

${ }^{12}$ K. W. Edmonds, N. R. S. Farley, R. P. Campion, C. T. Foxon, B. L. Gallagher, T. K. Johal, G. van der Laan, M. MacKenzie, J. N. Chapman, and E. Arenholz, Appl. Phys. Lett. 84, 4065 (2004)

${ }^{13}$ K. W. Edmonds, N. R. S. Farley, T. K. Johal, G. van der Laan, R. P. Campion, B. L. Gallagher, and C. T. Foxon, Phys. Rev. B 71, 064418 (2005)

${ }^{14}$ P. R. Stone, M. A. Scarpulla, R. Farshchi, I. D. Sharp, E. E. Haller, O. D. Dubon, K. M. Yu, J. W. Beeman, E. Arenholz, J. D. Denlinger, and H. Ohldag, Appl. Phys. Lett. 89, 012504 (2006).

${ }^{15}$ K. W. Edmonds, G. van der Laan, A. A. Freeman, N. R. S. Farley, T. K. Johal, R. P. Campion, C. T. Foxon, B. L. Gallagher, and E. Arenholz, Phys. Rev. Lett. 96117207 (2006).

${ }^{16}$ B. T. Thole, C. Carra, F. Sette, and G. van der Laan, Phys. Rev. Lett. 68, 1943 (1992).

${ }^{17}$ C. Carra, B. T. Thole, M. Altarelli, and X. Wang, Phys. Rev. Lett. 70, 694 (1993).

${ }^{18}$ L. Bergqvist, P. A. Korzhavyi, B. Sanyal, S. Mirbt, I. A. Abrikosov, L. Nordstrom, E. A. Smirnova, P. Mohn, P. Svedlindh, and O. Eriksson, Phys. Rev. B 67, 205201 (2003).

${ }^{19}$ A. W. Rushforth, N. R. S. Farley, R. P. Campion, K. W. Edmonds, C. R. Staddon, C. T. Foxon, B. L. Gallagher, and K. M. Yu, Phys. Rev. B 78, 085209 (2008).

This document was prepared as an account of work sponsored by the United States Government. While this document is believed to contain correct information, neither the United States Government nor any agency thereof, nor The Regents of the University of California, nor any of their employees, makes any warranty, express or implied, or assumes any legal responsibility for the accuracy, completeness, or usefulness of any information, apparatus, product, or process disclosed, or represents that its use would not infringe privately owned rights. Reference herein to any specific commercial product, process, or service by its trade name, trademark, manufacturer, or otherwise, does not necessarily constitute or imply its endorsement, recommendation, or favoring by the United States Government or any agency thereof, or The Regents of the University of California. The views and opinions of authors expressed herein do not necessarily state or reflect those of the United States Government or any agency thereof or The Regents of the University of California. 


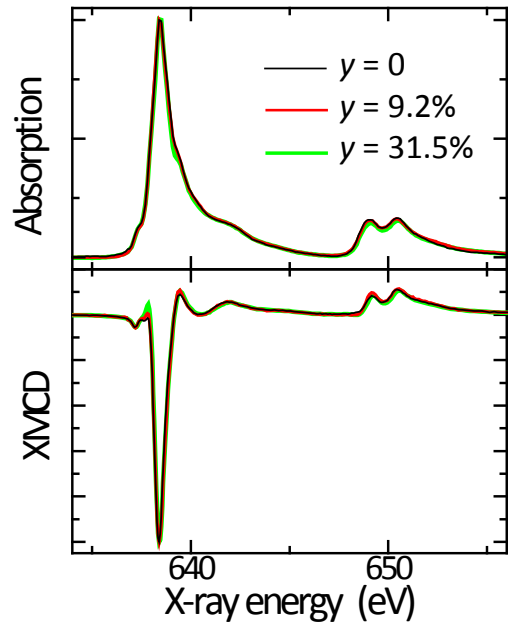

Figure 1. (Color online) $M n L_{2,3} x$-ray absorption spectra (top) and XMCD spectra (bottom) for the annealed $(\mathrm{Ga}, \mathrm{Mn})(\mathrm{As}, \mathrm{P})$ films with estimated $\mathrm{P}$ concentration $0 \%, 9.2 \%$ and $31.5 \%$.
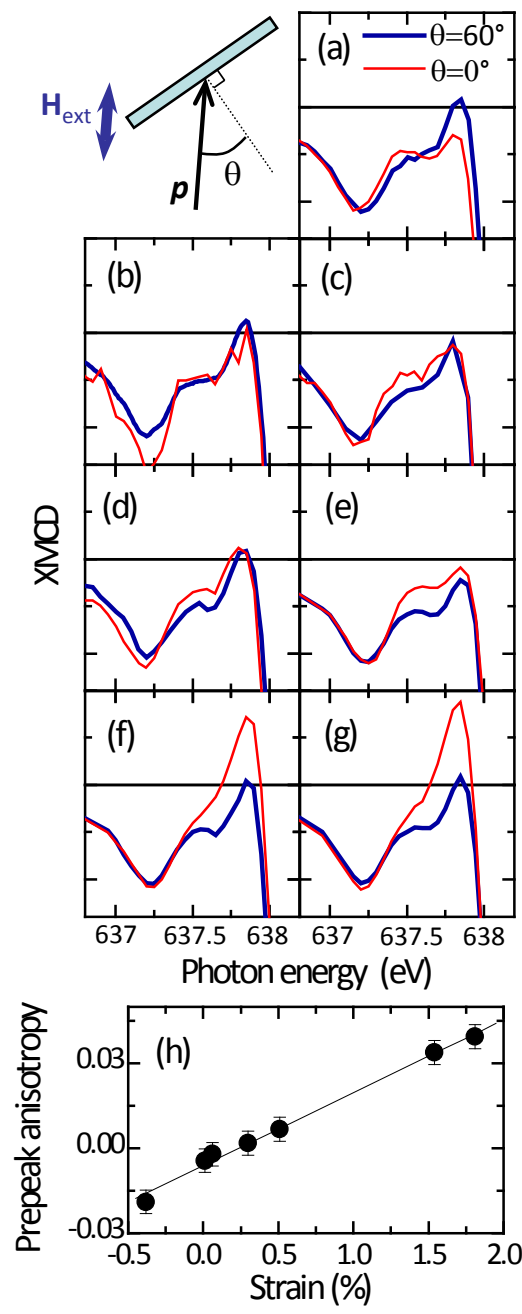

Figure 2. (Color online) (a-g) $M n L_{3}$ pre-edge regions of the XMCD spectra for the annealed $(\mathrm{Ga}, \mathrm{Mn})(\mathrm{As}, \mathrm{P})$ films with estimated $P$ concentration (a) $0 \%$, (b) $5.5 \%$, (c) $5.9 \%$, (d) $9.2 \%$, (e) $12.4 \%$, (f) $27.6 \%$, (g) $31.5 \%$. Thin lines are for normal incidence and thick lines are for $60^{\circ}$ incidence. The experimental geometry is illustrated in the top left of the figure. (h) Prepeak anisotropy (difference in XMCD intensity at $637.8 \mathrm{eV}$ ) versus epitaxial strain.

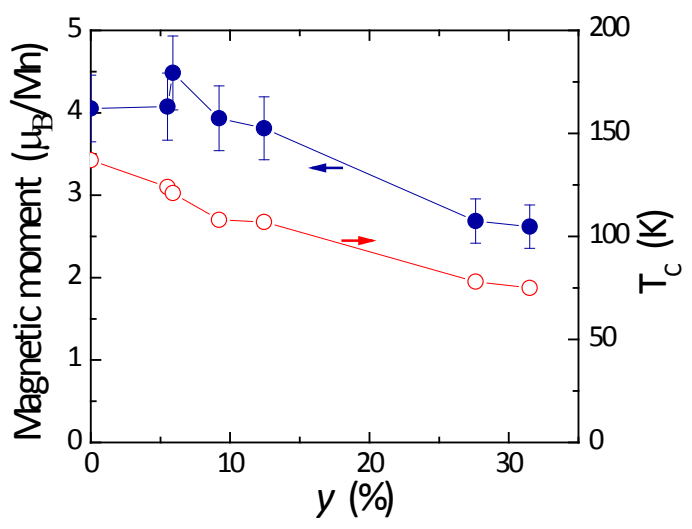

Figure 3. (Color online) Total magnetic moment per Mn ion obtained by XMCD sum rules (filled symbols, left axis) and $T_{C}$ obtained by SQUID magnetometry (open symbols, right axis), versus estimated $P$ concentration $y$. 